\title{
Post-transplant complications associated with gut flora
}

New data have revealed that kidney transplant recipients undergo changes in gut microbiota that might lead to post-transplant complications, including diarrhoea, urinary tract infections (UTIs) and acute rejection.

"Organ graft recipients receive multiple immunosuppressive drugs and antibiotics, and these agents are capable of altering the gut microbiota," explains lead author John R. Lee. "Gut microbial compositions are associated with infectious complications in stem-cell transplant recipients and perhaps with graft-versus-host disease, both of which served as some of the stimuli for our studies."

The microbial content of serial faecal specimens from kidney transplant recipients in the first 3 months posttransplantation was analysed using $16 \mathrm{~S}$ ribosomal RNA deep sequencing. A significant increase in the mean faecal abundance of Proteobacteria was apparent post-transplantation. Furthermore, patients with post-transplantation diarrhoea had significantly lower microbial diversity, with a notable absence of Bacteroides, Ruminococcus, Coprococcus and Dorea. The researchers suggest these findings could indicate an important function of these bacteria in the healthy gut.

Transplant recipients experiencing post-transplantation Enterococcus UTIs had a significantly elevated abundance of faecal Enterococcus, which corroborates with other published reports of faecal Enterococcus and post-transplantation infectious complications. In transplant

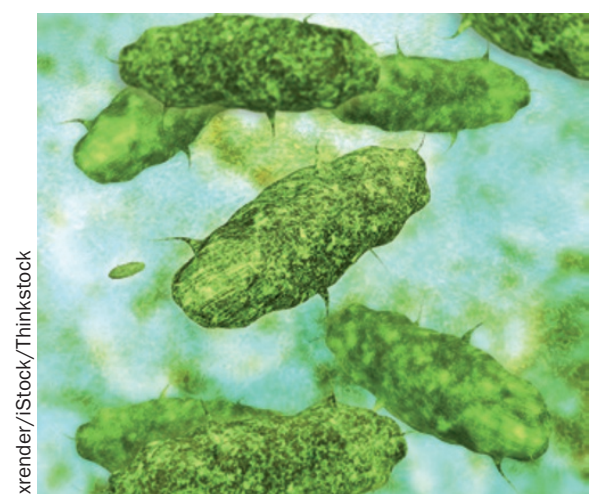

recipients who developed acute rejection, principal coordinate analyses revealed a microbial composition signature that was different from transplant recipients without acute rejection. In particular, patients with acute rejection had a greater abundance of Lactobacillales, Enterococcus, Anaerofilum and Clostridium tertium, and lower levels of Clostridiales, Bacteroidales and Lachnospiraceae.

When asked about future directions of this work, Lee adds: "We plan to validate the identified associations between the gut microbiota and post-transplantation complications in a larger group of kidney graft recipients."

"Ultimately, we plan to examine modulation of the gut microbiota as a preventative and/or therapeutic strategy in organ graft recipients," Lee concludes.

Peter Sidaway

Original article Lee, J. R. et al. Gut microbial community structure and complications after kidney transplantation: a pilot study. Transplantation doi:10.1097/TP.0000000000000370 